\title{
PESO CORPORAL, ÍNDICES CARDÍACOS, HEMATOCRITO Y HEMOGLOBINA EN DOS LÍNEAS COMERCIALES DE PAVOS
}

\author{
Body Weight, Cardiac Index Values, and Hematocrit and Haemoglobin \\ LeVels in Two Turkey Commercial Lines
}

\author{
Verónica K. Terry M. ${ }^{1}$, Edgardo Figueroa T. ${ }^{1,2}$, Nadia E. Fuentes N. ${ }^{1}$, \\ Milder Ayón S. ${ }^{3}$, Jhon Guzmán G. ${ }^{4}$, Fernando Carcelén C. ${ }^{5}$
}

\section{RESUMEN}

El presente estudio tuvo como objetivo comparar el peso corporal, así como índices cardiacos, hematocrito y hemoglobina como indicadores de trabajo cardiaco de dos líneas de pavos de engorde criados a nivel del mar. Se trabajó con 35 pavos de la línea BUT y 35 de la línea Hybrid. El peso corporal (PC) se registró desde la 4a a la 14ª semana de edad en que fueron beneficiados. En ese momento, se tomó una muestra de sangre para determinar el hematocrito y la hemoglobina; además, se extrajeron los corazones y se determinaron las relaciones de peso ventrículo derecho/ventrículo total (VD/VT), peso ventrículo izquierdo/ventrículo total (VI/VT), peso ventrículo izquierdo + septo/ ventrículo total (VI+S/VT), VI/PC, VD/PC, VI+S/PC y VT/PC. El peso corporal y la hemoglobina en pavos BUT fueron superiores $(\mathrm{p}<0.05)$, mientras que el hematocrito fue mayor $(\mathrm{p}<0.05)$ en pavos de la línea Hybrid. No se encontraron diferencias de los índices VD/VT, VI/VT, (VI+S)/VT y VD/PC entre las dos líneas, pero se encontró valores superiores $(\mathrm{p}<0.05)$ para las relaciones VI/PC, $(\mathrm{VI}+\mathrm{S}) / \mathrm{PC}$ y VT/PC en la línea BUT, concordante con la ganancia de peso.

Palabras clave: índice cardíaco, peso corporal, pavos, línea BUT, línea Hybrid, hematocrito, hemoglobina

\section{Abstract}

The study aimed to compare the body weight, and cardiac indices, hematocrit and hemoglobin as indicators of cardiac activity of two turkey commercial lines reared at sea level. Thirty five turkeys from each line were used in the study. Body weight (PC) was recorded from the $4^{\text {th }}$ to the $14^{\text {th }}$ week of age where birds were slaughtered. There, blood samples were collected for measuring hematocrit and hemoglobin levels. Also, hearts were collected to determine the following ratios: weight of right ventricle/total ventricle (VD/VT), weight of left ventricle/total ventricle (VI/VT), weight of left ventricle + septum/

\footnotetext{
${ }^{1}$ Laboratorio de Producción Avícola y Especies Menores, ${ }^{3}$ Laboratorio de Fisiología Animal, ${ }^{4}$ Laboratorio de Patología Aviar, ${ }^{5}$ Laboratorio de Bioquímica, Nutrición y Alimentación Animal, Facultad de Medicina Veterinaria, Universidad Nacional Mayor de San Marcos, Lima

${ }^{2}$ E-mail: edgardoft@gmail.com
} 
total ventricle (VI+S/VT), VI/PC, VD/PC, VI+S/PC, and VT/PC. Body weight and haemoglobin in BUT turkeys were higher $(\mathrm{p}<0.05)$ and hematocrit was higher $(\mathrm{p}<0.05)$ in Hybrid turkeys. No statistical differences were found on VD/VT, VI/VT, (VI+S)/VT, and $\mathrm{VD} / \mathrm{PC}$ between lines, but higher values were observed $(\mathrm{p}<0.05)$ for $\mathrm{VI} / \mathrm{PC}$, $(\mathrm{VI}+\mathrm{S}) / \mathrm{PC}$, and VT/PC in BUT turkeys, compatible with body weight gain.

Key words: cardiac index, body weight, turkey, BUT line, Hybrid line, hematocrit, haemoglobin

\section{INTRODUCCIÓN}

Los pavos que se crían en explotaciones industriales son mayormente productos de cruces genéticos programados para la obtención de animales con características productivas ventajosas. Estos cruzamientos son los denominados «híbridos comerciales» o líneas genéticas (Gura, 2007). En el Perú se explota principalmente las líneas de engorde Hybrid y BUT, cuya población se concentra en el departamento de Lima (Ciriaco, 2007).

La crianza de aves permite en la actualidad el beneficio de los animales a una edad más temprana, debido a un aumento en la velocidad de crecimiento y, por consiguiente, en el aumento de la masa muscular. Estos logros se deben a los avances genéticos (López et al., 1991) y mejoras en la nutrición, salud, manejo y ambiente. Sin embargo, el incremento en la velocidad de crecimiento trae como consecuencia el aumento en la carga metabólica del ave, lo que incrementa la demanda de oxígeno del organismo, demanda que no siempre puede ser compensada por los sistemas respiratorio y cardiovascular (Aziz, 2002).

La demanda adicional de oxígeno en el ave origina una secuencia de eventos que se inicia con un estado de hipoxia tisular que ocasiona un incremento del hematocrito y, con ello, el aumento de la viscosidad sanguínea. Esto puede desencadenar un cuadro de hipertensión arteriolar pulmonar, que puede ocasionar una hipertrofia cardiaca derecha y una serie de eventos que culminan con la muerte del animal por insuficiencia cardiaca congestiva (Wideman et al., 1998; González et al., 2000). Esta situación ha sido bastante estudiada en pollos de engorde (López et al., 1991).

El presente estudio tuvo como objetivo comparar el peso, así como el índice cardiaco, hematocrito y hemoglobina como indicadores de trabajo cardiaco corporal de dos líneas de pavos de engorde criados a nivel del mar, considerando que una alteración de estos valores podría derivar en la presentación del síndrome ascítico, muy similar a lo que sucede en los pollos parrilleros.

\section{MATERIALES y MéTODOS}

La crianza de los pavos se realizó en el galpón experimental del Laboratorio de Producción Avícola y Especies Menores y los análisis de laboratorio clínico en los laboratorios de Fisiología Animal y de Patología Aviar de la Facultad de Medicina Veterinaria de la Universidad Nacional Mayor de San Marcos, Lima.

Se trabajó con 35 pavos machos de la Línea BUT, elegidos al azar de un lote de 240 animales, y con 35 pavos machos de la Línea Hybrid elegidos al azar de un lote de 600 animales. Los pavos fueron marcados con un color por línea y criados junto con el lote al que pertenecían, empleando los estándares establecidos para las explotacio- 
nes comerciales en lo que respecta a manejo y sanidad. Se vacunaron contra la Enfermedad de Newcastle a los 19 días de edad y contra la viruela aviar a los 53 días de edad. La ración alimenticia incluyó insumos de disponibilidad local que cubrían las necesidades nutricionales de las aves (3.18\% de energía metabolizable y $20 \%$ de proteína total).

El pesaje de los animales se hizo a partir de la cuarta semana de edad y hasta la semana 14 , y siempre a la misma hora, empleando una balanza con capacidad de $30 \mathrm{~kg}$ y precisión de $10 \mathrm{~g}$. El beneficio de los animales se hizo a las 14 semanas de edad. Se colectaron muestras de sangre de cada animal en tubos de vidrio conteniendo heparina sódica $(200 \mathrm{UI} / \mathrm{ml})$ para las determinaciones de hematocrito y hemoglobina. El hematocrito se determinó por el método del microhematocrito con tubos capilares, centrifugados a 12000 rpm durante 5 minutos. La hemoglobina se determinó por el método de la cianometahemoglobina con el reactivo de Drabkin (Tietz, 1974).

Se colectaron los corazones para obtener los pesos cardíacos ventriculares. Para esto, se removió la grasa pericárdica, las aurículas y las válvulas cardíacas, y se procedió a determinar la relación VD/VT (peso del ventrículo derecho dividido entre el peso de la masa ventricular total), utilizando el protocolo descrito por Alexander y Jensen (1959). Además, se determinó la relación ventrículo derecho/peso corporal (VD/PC) para establecer el desarrollo del ventrículo derecho con relación al desarrollo corporal. Otros índices cardiacos evaluados fueron: VI/ VT (ventrículo izquierdo/ventrículo total), VI+S/VT (ventrículo izquierdo + septo / ventrículo total), VI/PC (Ventrículo izquierdo/peso corporal), VI+S/PC (ventrículo izquierdo + septo / peso corporal) y VT/PC (ventrículo total/peso corporal).

La variable de peso vivo semanal fue analizada mediante un análisis de varianza de dos vías, teniendo como factores la se- mana de vida y la línea comercial. Las variables peso vivo en la semana 14, índices cardiacos, hematocrito y hemoglobina, por proceder de un mismo animal, fueron analizadas mediante un análisis multivariado de varianza, teniendo como variable explicativa a la línea comercial. Se realizó la prueba de Chi cuadrado para determinar diferencias entre líneas con respecto a la mortalidad por insuficiencia cardíaca congestiva con ascitis e hidropericardio. Los análisis estadísticos se llevaron a cabo mediante el uso del software SPSS 15.0 para Windows.

\section{Resultados y Discusión}

El peso corporal en la semana 14 fue significativamente mayor en pavos BUT que en aquellos de la línea Hybrid $(\mathrm{p}<0.05)$. El peso entre las dos líneas no fue significativamente diferente entre las semanas 4 y 8 de edad, pero luego, las aves de la línea BUT tuvieron un mayor peso corporal hasta la semana $14(\mathrm{p}<0.05)$, con excepción de la semana 13 (Fig. 1).

El mayor peso corporal de los pavos BUT exige una mayor tasa metabólica, ya que son aves de rápido crecimiento y requieren un alto consumo de oxígeno; sin embargo, las dos líneas de aves son consideradas como medianamente pesadas en comparación con la línea Nicholas (Ciriaco, 2007). Las capacidades cardiovascular y metabólica de los pavos están considerablemente influenciadas por el procedimiento de selección para la producción de carne (Romvári et al., 2004; Sherwin, 2005). Los pavos de las líneas BUT y Hybrid, de acuerdo a parámetros productivos estándares en el país, pesan entre $11.1 \mathrm{a} 11.7 \mathrm{~kg}$ a las 14 semanas de edad (Quintana, 1991; Ciriaco, 2007). Los machos pesan más que las hembras (Scholtyssek, 1970). Sin embargo, los pesos obtenidos en el presente trabajo fueron menores a lo establecido en ambas líneas, posiblemente por efecto del ambiente, manejo y alimentación. 


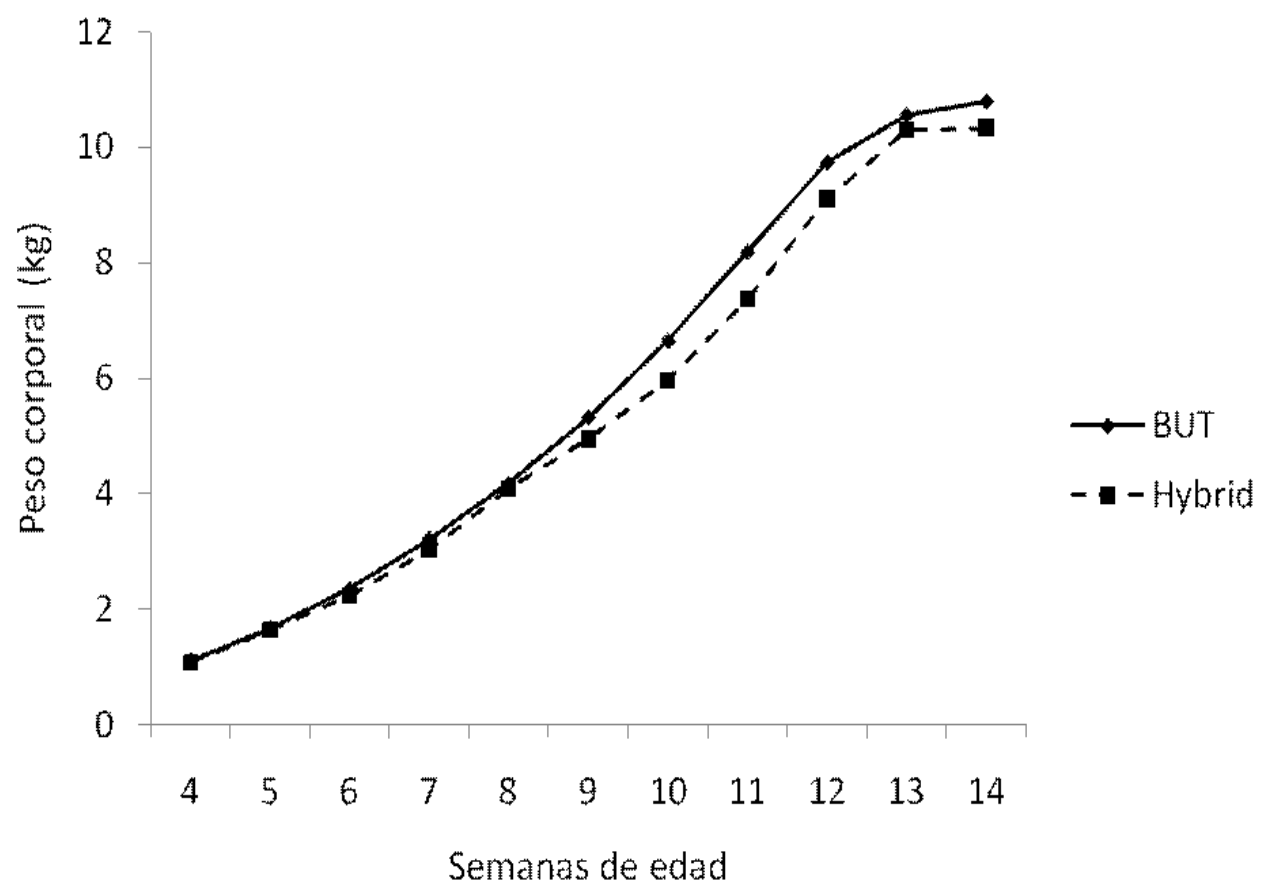

Figura 1. Curva de crecimiento (kg) de pavos machos de las líneas comerciales BUT (n=35) e Hybrid ( $\mathrm{n}=35$ )

La hemoglobina fue mayor $(\mathrm{p}<0.05)$ en pavos BUT pero el hematocrito fue mayor en pavos Hybrid $(\mathrm{p}<0.05)$. No se encontraron diferencias significativas entre las relaciones VD/VT, VI/VT, (VI+S)/VT y VD/PC entre líneas, pero hubo diferencia estadística $(\mathrm{p}<0.05)$ en las relaciones de $\mathrm{VI} / \mathrm{PC}$, $(\mathrm{VI}+\mathrm{S}) / \mathrm{PC}$ y $\mathrm{VT} / \mathrm{PC}$.

Los valores de hematocrito y hemoglobina observados en el estudio concuerdan con los valores normales descritos para pavos domésticos (Schales C y Schales K, 1994; Bounous et al., 2000). Un alto valor de hematocrito implica un aumento de la viscosidad de la sangre y de resistencia al flujo sanguíneo, causando una mayor carga de trabajo al corazón (Ploog, 1982, Hernández, 1986; Shlosberg et al., 1996), condición que unida a la demanda de sangre oxigenada por los órganos del cuerpo, hace que se incremente el ritmo y gasto cardiaco en aves sometidas a hipoxia (Ploog, 1982) o en aves de elevado metabolismo celular, condiciones que no se observaron en las aves de este experimento.

Las relaciones de VD/VT, VI/VT, $\mathrm{VI}+\mathrm{S} / \mathrm{VT}$ y VD/PC fueron similares en las dos líneas de pavos, posiblemente debido a que estas aves no desarrollaron toda su capacidad de ganancia de peso corporal durante el estudio (Ciriaco, 2007); sin embargo, los valores ventriculares en relación al peso corporal (VI/PC, VI+S/PC, VT/PC) fueron mayores en pavos BUT ( $\mathrm{p}<0.05$, Cuadro 1$)$. Estos resultados son el reflejo del metabolismo y la elevada demanda de oxígeno (Moreno y Hernández, 1996). En condiciones normales de tensión de oxígeno, la mayor ganancia de peso corporal coincide con un mayor incremento del gasto cardiaco (Sabán, 2009; Zhang y Reisen, 2000). 
Cuadro 1. Valores de hematocrito, hemoglobina e índices cardía $\cos ^{1}$, expresados en promedio \pm desviación estándar, de pavos de las líneas BUT e Hybrid entre la $4^{\mathrm{a}}$ y $14^{\mathrm{a}}$ semana de edad criados a nivel del mar

\begin{tabular}{lcc}
\hline & Línea BUT & Línea Hybrid \\
\hline Hematocrito (\%) & $34.80 \pm 1.76^{\mathrm{b}}$ & $36.25 \pm 2.45^{\mathrm{a}}$ \\
Hemoglobina (g/100 ml) & $14.35 \pm 0.81^{\mathrm{a}}$ & $13.39 \pm 0.71^{\mathrm{b}}$ \\
VD/VT & $0.15 \pm 0.01^{\mathrm{a}}$ & $0.16 \pm 0.01^{\mathrm{a}}$ \\
VI/VT & $0.61 \pm 0.02^{\mathrm{a}}$ & $0.60 \pm 0.03^{\mathrm{a}}$ \\
$(\mathrm{VI}+\mathrm{S}) / \mathrm{VT}$ & $0.84 \pm 0.01^{\mathrm{a}}$ & $0.84 \pm 0.01^{\mathrm{a}}$ \\
VD/PC & $0.40 \pm 0.04^{\mathrm{a}}$ & $0.38 \pm 0.04^{\mathrm{a}}$ \\
VI/PC & $1.65 \pm 0.22^{\mathrm{a}}$ & $1.50 \pm 0.27^{\mathrm{b}}$ \\
(VI+S)/PC & $2.26 \pm 0.26^{\mathrm{a}}$ & $2.08 \pm 0.31^{\mathrm{b}}$ \\
VT/PC & $2.67 \pm 0.30^{\mathrm{a}}$ & $2.46 \pm 0.35^{\mathrm{b}}$ \\
\hline a,b & Superíndices diferentes dentro de cada parámetro indican diferencias estadísticas (p<0.05) \\
VD: ventrículo derecho; VI: ventrículo izquierdo; VT: los dos ventrículos; S: septo interventricular \\
PC: peso corporal
\end{tabular}

\section{Conclusiones}

- El peso corporal al final del periodo de crianza fue mayor $(\mathrm{p}<0.05)$ en pavos de la línea BUT que en los de la línea HYBRID.

- Las aves de la línea BUT tuvieron mayores valores $(\mathrm{p}<0.05)$ en los índices VI/ $\mathrm{PC}, \mathrm{VI}+\mathrm{S} / \mathrm{PC}$ y VT/PC que la línea Hybrid. Así mismo, no hubo diferencia en los índices VD/VT, VI/VT, (VI+S)/ VT y VD/PC entre las dos líneas.

- El valor del hematocrito fue mayor en la línea Hybrid y el valor de la hemoglobina fue mayor en la línea BUT; sin embargo, en ambos casos, los valores se encuentran dentro de los rangos de normalidad de la especie.

\section{Literatura Citada}

1. Alexander AF, Jensen R. 1959. Gross cardiac changes in cattle with high mountain (brisket) disease and in experimental cattle maintained at high altitude. Am J Vet Res 20: 680-689.
2. Aziz T. 2002. Round-heart disease, a common turkey affliction. World Poultry 18(12): 41-42.

3. Bounous DI, Wyatt RD, Gibbs PS, Kilburn JV, Quist CF. 2000. Normal hematologic and serum biochemical reference intervals for juvenile wild turkeys. J Wildlife Dis 36: 393-396.

4. Ciriaco PC. 2007. Engorde de pavos. Programa de investigación en avicultura. Lima: Facultad de Zootecnia, UNALM. 60 p.

5. González JM, Suárez ME, Pró A, López C. 2000. Restricción alimenticia y salbutamol en el control del síndrome ascítico en pollo de engorda: 2 . Respuesta hematológica y cardiaca. Agrociencia 34: 293-301.

6. Gura S. 2007. Empresas de genética ganadera: Estrategias de apropiación y concentración de un poder emergente en la economía alimentaria mundial. OberRamstadt, Alemania: Livestock Genetics Companies. 32 p.

7. Hernández VA. 1986. La ascitis hipóxica en pollos: influencia y posibles soluciones. Avicultura Prof 4:152-153. 
8. López C, Arce J, Ávila E, Vásquez C. 1991. Investigaciones sobre el síndrome ascítico en pollos de engorda. Ciencia Vet 5: 13-48.

9. Moreno M, Hernández A. 1996. La hipertensión pulmonar en pollos de engorde. Patogénesis, control y perspectivas de investigación. CEISA 3(1): 65-79.

10. Ploog HP. 1982. Efecto de la altura en pollos broilers. Ovonoticias 48: 48-51.

11. Quintana JA. 1991. Avitecnia - Manejo de las aves domésticas. $2^{\mathrm{a}}$ ed. México: Ed Trillas. 305 p.

12. Romvári R, Petrási $Z$, Sütõ $Z$, Szabó A, Andrássy G, Garamvölgyi R, Horn $P$. 2004. Noninvasive characterization of the turkey heart performance and its relationship to skeletal muscle volume. Poultry Sci 83: 696-700.

13. Sabán R. 2009. Control global del riesgo cardiometabólico. España: Ed Díaz de Santos. 920 p.

14. Schales C, Schales K. 1994. Galliformes. In: Ritchie BW, Harrison GJ, Harrison LR (eds). Avian Medicine: principles and application. Florida: Wingers Publ. p 1218-1236.
15. Scholtyssek S. 1970. Manual de avicultura moderna. Zaragoza: EdAcribia. 359p.

16. Sherwin CM. 2005. Turkeys: behaviour, management, and well-being. In: Pond WG, Bell AW (eds). Encyclopedia of animal science. USA: Marcel Dekker. p 847-849.

17. Shlosberg A, Bellaiche M, Zeitlin G, Ya'Acobi M, Cahaner A. 1996. Hematocrit values from ascites in coldstressed broilers from parents selected by hematocrit. Poultry Sci 75: 1-5.

18. Tietz NW. 1976. Fundamentals of clinical chemistry. $2^{\text {nd }}$ ed. Philadelphia: WB Saunders. $411 \mathrm{p}$.

19. Wideman RF, Wing T, Kirby YK, Forman MF, Marson N, Tackett CD, Ruiz-Feria CA. 1998. Evaluation of minimally invasive indices for predicting ascites susceptibility in three successive hatches of broilers exposed to cool temperatures. Poultry Sci 77: 1565-1573.

20. Zhang R, Reisen E. 2000. Obesityhypertension: The effects on cardiovascular and renal system. Am J Hypertens 13: 1308-1314. 